\title{
New polylactide/layered silicate nanocomposites. 5. Designing of materials with desired properties
}

\author{
Suprakas Sinha Ray ${ }^{\mathrm{a}}$, Kazunobu Yamada ${ }^{\mathrm{b}}$, Masami Okamoto ${ }^{\mathrm{a}, *}$, Youhei Fujimoto ${ }^{\mathrm{a}}$, \\ Akinobu Ogami ${ }^{\text {b }}$, Kazue Ueda ${ }^{\mathrm{b}}$ \\ ${ }^{a}$ Advanced Polymeric Materials Engineering, Graduate School of Engineering, Toyota Technological Institute, Hisakata 2-12-1, Tempaku, \\ Nagoya 468 8511, Japan \\ ${ }^{\mathrm{b}}$ Unitika Ltd, Kozakura 23, Uji, Kyoto 6610021 , Japan
}

Received 24 February 2003; received in revised form 23 June 2003; accepted 5 August 2003

\begin{abstract}
Understanding the structure/property relationship in polymer/layered silicate nanocomposites is of great importance in designing materials with desired properties. In order to understand these relations, a series of polylactide (PLA)/organically modified layered silicate (OMLS) nanocomposites have been prepared using a simple melt extrusion technique. Four different types of OMLS have been used for the preparation of nanocomposites, three were modified with functionalized ammonium salts while fourth one was a phosphonium salt modified OMLS. The structure of the nanocomposites in the nanometer scale was characterized by using wide-angle X-ray diffraction and transmission electron microscopic observations. Using four different types of layered silicates modified with four different types of surfactants, the effect of OMLS in nanocomposites was investigated by focusing on four major aspects: structural analysis, thermal properties and spherulite morphology, materials properties, and biodegradability. Finally, we draw conclusions about the structure/property relationship in the case of PLA/OMLS nanocomposites.
\end{abstract}

(C) 2003 Elsevier Ltd. All rights reserved.

Keywords: Polylactide; Nanocomposites; Materials properties

\section{Introduction}

Over the last few years, the utility of inorganic nanoscale particles as filler to enhance the polymer performance has been established. Of particular interest are nanocomposites consisting of a polymer and organically modified layered silicate (OMLS) because they often exhibit remarkably improved materials properties as compared to those of pristine polymers containing small amount $(\leq 5 \mathrm{wt} \%)$ of layered silicate. These improvements can include high moduli [1-5], increased strength and heat resistance [1-5, $6]$, decreased gas permeability [1-5,7-11] and flammability [2,5,12-15], and increased biodegradability of biodegradable polymers [16-17]. In general, it is believed that these property improvements come from matrixreinforcement interactions present in nanocomposites as opposed to conventional composites (both micro- and

\footnotetext{
* Corresponding author. Tel.: + 81-52-809-1861; fax: + 81-52-809-1864.

E-mail address: okamoto@toyota-ti.ac.jp (M. Okamoto).
}

macro-composite). On the other hand, these materials have also been proved unique model systems to study the structure and dynamics of polymers in confined environments $[4,18,19]$.

Layered silicates have individual layer thickness in the order of $1 \mathrm{~nm}$ and very high aspect ratios (10-1000). A few weight percent of OMLS that are properly distributed throughout the polymer matrix thus create much higher surface area for polymer-filler interaction than do conventional composites [20].

Depending on the strength of the polymer/OMLS interfacial interactions structurally three broad classes of nanocomposites are thermodynamically achievable (see Fig. 1):

(a) Intercalated nanocomposites, in which insertion of polymer chains into the layered silicate structure occurs in a crystallographically regular fashion, regardless of the OMLS to polymer ratio, and a repeat distance of few nanometers. 


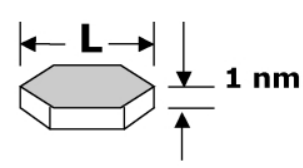

One layered silicate Platelet L: $100-200 \mathbf{n m}$ in case of MMT

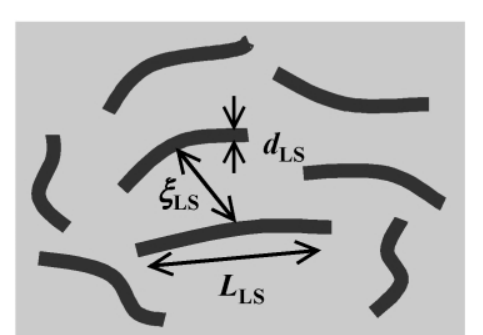

Form factors of dispersed layered silicate

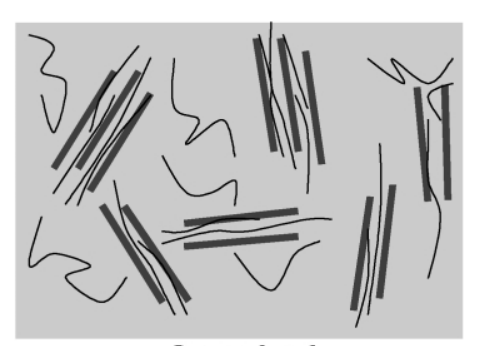

Intercalated

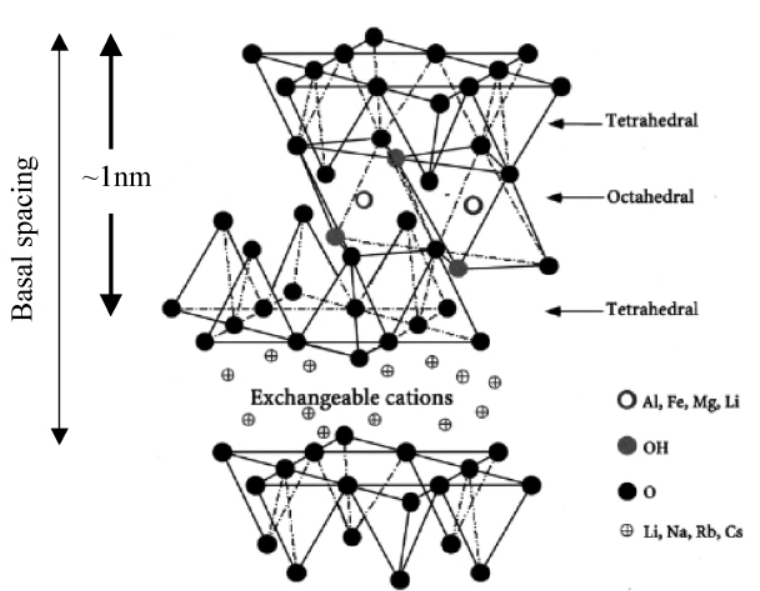

The structure of 2:1 layered silicates

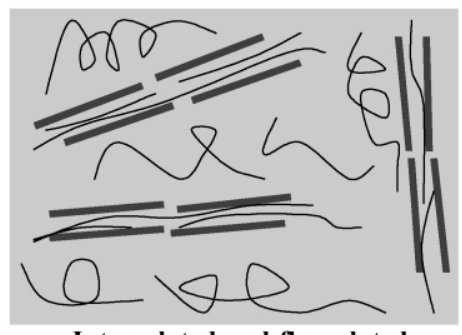

Intercalated-and-flocculated

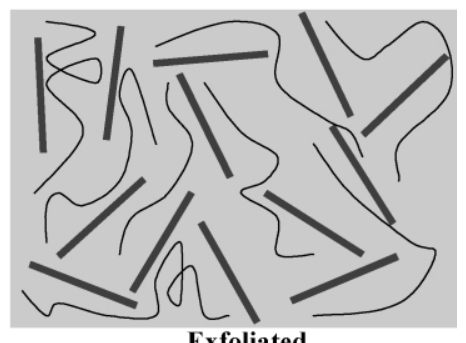

Exfoliated

Fig. 1. Schematic illustrations of three broad classes of thermodynamically achievable polymer/layered silicate nanocomposites.

(b) Flocculated nanocomposites, conceptually same with the intercalated nanocomposites, however, stacked and intercalated silicate layers some time flocculated due to the hydroxylated edge-edge interaction of the silicate layers.

(c) Exfoliated nanocomposites, in an exfoliated nanocomposite, the individual silicate layers are separated in a continuous polymer matrix by an average distances that totally depends on OMLS loading. Usually, the OMLS content of an exfoliated nanocomposite is much lower than that of intercalated nanocomposite.

Most of the nanocomposite researchers believe that the preparation of completely exfoliated structure is the ultimate target for better overall properties. However, these significant improvements are not observed in every nanocomposite systems, including systems where the silicate layers are completely exfoliated [20]. While from the barrier property standpoint, the development of exfoliated nanocomposites is preferred always. On the other hand, Nylon-6/OMLS nanocomposite system is completely different from other nanocomposite systems because there is a very strong interaction between Nylon-6 matrix and silicate layers by the formation of strong hydrogen bonds [21]. This interaction leads to the better overall properties with exfoliated structure.

Generally, intercalation of polymer chains into the silicate galleries is done by using one of the following two approaches: (1) insertion of suitable monomers in the silicate galleries and subsequent polymerization [22-25] or (2) direct insertion of polymer chains into the silicate galleries from either solution [26-27] or the melt [28]. Since the possibility of direct melt intercalation was first demonstrated by Vaia et al. [28], recently the melt intercalation technique has become a main stream for the preparation of polymer/OMLS nanocomposites because it is quite suitable for industries uses [29-32]. This process involves annealing, statically or under shear, a mixture of polymer and OMLS above the softening point of the polymer [33]. During anneal the polymer chains diffuse from the bulk polymer melt into the galleries between the silicate layers.

Recently, materials based on biodegradable polymers with excellent properties are the subjects of active research attention worldwide. One of the most promising candidates in this direction is PLA. PLA is linear aliphatic thermoplastic polyester, produced from renewable sources with excellent properties comparable to many petroleum-based plastics and readily biodegradable $[34,35]$. High-molecularweight PLA is generally produced by the ring-opening polymerization of lactide [36-39]. Lactide is a cyclic dimer prepared by the controlled depolymerization of lactic acid, which in turn is obtained from the fermentation of sugar feedstocks, corn, etc. [40]. PLA has good mechanical properties, thermal plasticity and biocompatibility, and is readily fabricated, thus being a promising polymer for various end-use applications [41]. Currently, there is increasing interest in using PLA for disposable degradable 
plastic articles [42] However, other properties of PLA such as flexural properties, heat distortion temperature (HDT), gas permeability, impact factor, melt viscosity for further processing, etc. are often not good enough for various enduse applications [43]. Accordingly, we have planned to design environmentally benign nanocomposites based on PLA and OMLS that have mechanical and various other materials properties suitable for wide-range of applications. On the other hand, layered silicates are naturally abundant, economic, and more importantly benign to the environment.

We have previously reported [16-17,44-49] on the preparation, characterization, mechanical and materials properties of various PLA/OMLS nanocomposites. The mechanical and materials properties of pure PLA were concurrently improved by the introduction and intercalation of OMLS, using a melt extrusion technique.

The present work first explores the internal structure of a series of PLA/OMLS nanocomposites prepared with four different types of OMLS using a simple melt extrusion. Secondly, effect of OMLS on thermal properties and crystallization behavior of pure PLA. Subsequently, we focus on a comparative study of the mechanical and flexural properties, oxygen $\left(\mathrm{O}_{2}\right)$ gas permeability and biodegradability of pure PLA and various nanocomposites. Finally, we try to make conclusions on the structure/property relationship in the case of PLA/OMLS nanocomposites.

\section{Experimental part}

\subsection{Materials}

PLA with a D-lactide content of $1.1-1.7 \%$ (supplied by Unitika Co. Ltd) was dried under vacuum at $60{ }^{\circ} \mathrm{C}$ and then kept under dry nitrogen gas for one week prior to use. The four different types of OMLS used in this study were synthesized by replacing $\mathrm{Na}^{+}$ions in different layered silicates with alkylammonium or alkylphosphonium cations by ion exchange. Table 1 summarized the detail specifications and abbreviation (used in the text) of four different types of OMLS used in this research.

\subsection{Nanocomposite preparation}

Nanocomposites were prepared by melt extrusion. OMLS (powder form) and PLA (pellets form) were first dry-mixed by shaking them in a bag. The mixture was then melt extruded using a twin-screw extruder (PCM-30, Ikegai machinery Co.) operated at $210^{\circ} \mathrm{C}$ (screw speed $=100$ $\mathrm{rpm}$, feed rate $=120 \mathrm{~g} / \mathrm{min}$ ) to yield nanocomposite strands. ${ }^{1}$ The abbreviations of various nanocomposites

\footnotetext{
${ }^{1}$ We have checked the degradation of various types of intercalated salts in four different types of OMLS using thermogravimetric analysis. Up to $210^{\circ} \mathrm{C}$, there is almost no degradation of intercalated salts in all types of OMLS.
} 
prepared using four different types of OMLS (each containing $4 \mathrm{wt} \%$ of OMLS) are also presented in Table 1. The extruded strands were pelletized and dried under vacuum at $60{ }^{\circ} \mathrm{C}$ for $48 \mathrm{~h}$ to remove water. The dried pellets were then converted into sheets with a thickness of $0.7-$ $2 \mathrm{~mm}$ by pressing with $\approx 1.5 \mathrm{MPa}$ at $190{ }^{\circ} \mathrm{C}$ for 3 min using a hot press. The molded sheets were then quickly quenched between glass plates and then annealed at $110^{\circ} \mathrm{C}$ for $1.5 \mathrm{~h}$ for various characterizations.

\subsection{Characterization}

\subsubsection{Wide-angle $X$-ray diffraction}

Wide-angle X-ray diffraction (WAXD) analyses were performed for four different types of pure OMLS powders and corresponding nanocomposites on MXlabo X-ray diffractometer (MAC Science Co., $3 \mathrm{~kW}$, a graphite monochromator, $\mathrm{Cu} \mathrm{K}_{\alpha}$ radiation with $\lambda=0.154 \mathrm{~nm}$ ), operated at $40 \mathrm{kV} / 20 \mathrm{~mA}$. Samples were scanned in fixed time (FT) mode with a counting time of $2 \mathrm{~s}$ under diffraction angle $2 \Theta$ in the range of $1^{\circ}-70^{\circ}$.

\subsubsection{Transmission electron microscopy}

Nanoscale structure of various nanocomposites was investigated by means of a high resolution Transmission electron microscopy (TEM) (H-7100, Hitachi Co.), operated at an accelerating voltage of $100 \mathrm{kV}$. The ultra thin sections (the edge of the sample sheet perpendicular to the compression mold) with a thickness of $100 \mathrm{~nm}$ were microtomed at $-80{ }^{\circ} \mathrm{C}$ using a Reichert Ultra cut cryoultramicrotome without staining.

\subsubsection{Gel permeation chromatography}

Number-average $\left(M_{\mathrm{n}}\right)$ and weight-average $\left(M_{\mathrm{w}}\right)$ molecular weights of PLA matrix before and after nanocomposites preparation were determined by means of Gel permeation chromatography (GPC) (LC-VP, Shimadzu Co.), using polystyrene standards for the calibration and tetrahydrofuran (THF) as the solvent at $40{ }^{\circ} \mathrm{C}$ with a flow rate of $0.5 \mathrm{ml} / \mathrm{min}$. For the GPC measurements first PLA or nanocomposites were dissolved in $\mathrm{CHCl}_{3}$ and then diluted with THF. $M_{\mathrm{w}}$ and PDI $\left(M_{\mathrm{w}} / M_{\mathrm{n}}\right)$ of PLA in pure state and in OMLS filled systems are summarized in Table 2. From Table 2, we can see that the incorporation of OMLS filler into the PLA matrix resulted in a reduction in the molecular weight of the matrix and this decrease little sharp in the case of MEE or SAP filled systems. Decreased molecular weights of nanocomposites may be explained by either the shears mixing of PLA and OMLS or the presence of modified salt, both resulting in a certain extent of hydrolysis at high temperature, and latter factor may vary from OMLS to OMLS. So molecular weight of PLA matrix after nanocomposites preparation completely depends upon the nature of salt used for the modification of pristine layered silicate.

\subsubsection{Thermal properties}

Glass transition $\left(T_{\mathrm{g}}\right)$, melting $\left(T_{\mathrm{m}}\right)$ and crystallization $\left(T_{\mathrm{c}}\right)$ temperatures as well as degree of crystallinity $\left(\chi_{\mathrm{c}}\right)$ of PLA before and after nanocomposites preparation were determined using a temperature-modulated differential scanning calorimeter (TMDSC) (MDSC ${ }^{\mathrm{TM}}$, TA2920, TA instruments), operated at a heating rate of $5 \% \mathrm{~min}$ with a heating/cooling cycle of the modulation period of $60 \mathrm{~s}$ and the amplitude of $\pm 0.769{ }^{\circ} \mathrm{C}$. For the measurement of $\chi_{\mathrm{c}}$, prior to the DSC analysis, the extra heat absorbed by the crystallites formed during heating had to be subtracted from the total endothermic heat flow due to the melting of the whole crystallites as was described previously by us [50]. By considering the melting enthalpy of $100 \%$ crystalline poly (L-lactide) as $93 \mathrm{~J} / \mathrm{gm}$ [51], $\chi_{\mathrm{c}}$ was estimated for pure PLA and various nanocomposites, and is summarized in Table 2 .

\subsubsection{Light scattering and polar optical micrographic observations}

Crystallite texture of pure PLA and various nanocomposite samples were investigated by means of Light scattering (LS) experiments under Hv- (cross-polarized) optical alignment mode with the radiation of polarized $\mathrm{He}-\mathrm{Ne}$ laser of $632.8 \mathrm{~nm}$ wavelengths. The details of the LS measurement were described in our previous paper [52].

Crystallite growth behavior of pure PLA and various nanocomposite samples was also investigated by means of polar optical micrograph (POM). Dried pellets were first sandwiched between two pieces of cover glass, placed on a laboratory hot plate at $190^{\circ} \mathrm{C}$ for $60 \mathrm{~s}$ to obtain a thin film of $\sim 30 \mu \mathrm{m}$ in thickness. The molten film was then rapidly quenched to the desired temperature $\left(110^{\circ} \mathrm{C}\right)$ by putting it on a thermostatted hot-stage (Linkam RTVMS, Linkam Scientific Instruments, Ltd) mounted on a POM (Nikon

Table 2

Characteristic parameters of pure PLA and various nanocomposites

\begin{tabular}{|c|c|c|c|c|c|c|c|}
\hline Samples & $M_{\mathrm{w}} \times 10^{-3}(\mathrm{~g} / \mathrm{mol})$ & $M_{\mathrm{w}} / M_{\mathrm{n}}$ & $T_{\mathrm{g}} /{ }^{\circ} \mathrm{C}$ & $T_{\mathrm{m}} /{ }^{\circ} \mathrm{C}$ & $T_{\mathrm{c}} /{ }^{\circ} \mathrm{C}$ & $\chi_{\mathrm{c}} / \%$ & $N \times 10^{5} /(\mu \mathrm{m})^{-1}$ \\
\hline PLA & 177 & 1.58 & 60.0 & 168.2 & 127.2 & 36.0 & 8 \\
\hline PLA/ODA4 & 161 & 1.58 & 59.3 & 169.8 & 98.5 & 49.1 & 100 \\
\hline PLA/SBE4 & 163 & 1.61 & 59.7 & 169.3 & 100.1 & 65.0 & 80 \\
\hline PLA/SAP4 & 149 & 1.60 & 56.0 & 168.1 & 89.7 & 39.7 & 3 \\
\hline PLA/MEE4 & 150 & 1.55 & 56.4 & 168.6 & 99.4 & 39.6 & 70 \\
\hline
\end{tabular}


OPTIPHOTO2-POL). After complete crystallization, the nature of spherulitic growth was observed using POM.

\subsubsection{Dynamic mechanical analysis (DMA)}

Dynamic mechanical properties of pure PLA and various nanocomposites were measured using a Reometrics Dynamic Analyzer (RDAII) in the tension-torsion mode. The temperature dependence of dynamic storage modulus $\left(G^{\prime}\right)$ and $\tan \delta$ of pure PLA and nanocomposites were measured at a constant frequency $(\omega)$ of $6.28 \mathrm{rad} / \mathrm{s}$, a strain amplitude of $0.05 \%$, and in the temperature range of $-20{ }^{\circ} \mathrm{C}$ to $160{ }^{\circ} \mathrm{C}$ with a heating rate of $2^{\circ} \mathrm{C} / \mathrm{min}$.

\subsubsection{Flexural properties and heat distortion test}

Dried pure PLA and nanocomposites pellets were injection-molded using an injection machine (IS-80G, Toshiba Machinery Co.) operated at $190{ }^{\circ} \mathrm{C}$ with a mold temperature of $30^{\circ} \mathrm{C}$. Flexural modulus and strength of the injection-molded specimens (thickness $\sim 3.2 \mathrm{~mm}$, annealed at $120{ }^{\circ} \mathrm{C}$ for $30 \mathrm{~min}$ ) were measured according to the ASTM D-790 method (Model 2020, Intesco Co.) with a strain rate of $2 \mathrm{~mm} / \mathrm{min}$ at room temperature. Heat distortion tests were conducted using annealed injectionmolded samples, (Heat Distortion Tester, Toyoseiki Co.) according to the ASTM D-648 method with a heating rate of $2{ }^{\circ} \mathrm{C} / \mathrm{min}$

\subsubsection{Measurement of $\mathrm{O}_{2}$ gas transmission rate}

$\mathrm{O}_{2}$ gas transmission rate of pure PLA and various nanocomposites were measured at $20{ }^{\circ} \mathrm{C}$ and $90 \%$ relative humidity by the ASTM D-1434 differential pressure method (GTR-30XAU, Yanaco Co.). Test samples were prepared by compression molding (thickness $\sim 300 \mu \mathrm{m}$ ), and melt quenched samples were used.

\subsubsection{Biodegradability}

Biodegradability was studied on a homemade compost instrument at $(58 \pm 2){ }^{\circ} \mathrm{C}$. Carbon dioxide $\left(\mathrm{CO}_{2}\right)$ evolution was measured directly by means of an attached FT-IR spectrometer (Horiba, FT-730). The compost used was prepared from the mixture of bean-curd refuse, food waste and cattle feces. The test specimens were crystallized (at $110{ }^{\circ} \mathrm{C}$ for $1.5 \mathrm{~h}$ ) pellets.

\section{Results and discussion}

\subsection{Nanocomposite structure}

Due to its easiness and availability WAXD is the most commonly used tool to probe the nanocomposite structure [1-5] and some times to study the kinetics of the polymer melt intercalation [53]. By monitoring the position, shape, and intensity of the basal reflections from the dispersed silicate layers, the nanocomposite structure either intercalated or exfoliated may be identified. For example, in case of exfoliated nanocomposites, the extensive layer separation associated with the delamination of the original silicate layers in the polymer matrix results in the eventual disappearance of any coherent X-ray diffraction from the distributed silicate layers. On the other hand, for intercalated nanocomposites, the finite layer expansion associated with the polymer intercalation results in the appearance of a new basal reflection corresponding to the larger gallery height.

Although, WAXD offers a convenient method to determine the interlayer spacing of the silicate layers in the original layered silicates and in the intercalated nanocomposites (within 1-4 nm), however, little can be said about the spatial distribution of the silicate layers or any structural inhomogeneities in the nanocomposites. Additionally, some layered silicates initially do not exhibit well-defined basal reflection. Thus, peak broadening and intensity decreases are very difficult to study systematically. Therefore, conclusions concerning the mechanism of nanocomposites formation and their structure based solely on WAXD patterns are only tentative. On the other hand, TEM allows a qualitative understanding of the internal structure, spatial distribution of the various phases, and defect structure through direct visualization. However, special care must be exercised to guarantee a representative cross section of the sample. Here we combine WAXD and TEM to determine the structure of various nanocomposites prepared using a simple melt extrusion technique. The intercalation of polymer chains into the silicate galleries usually increases the interlayer spacing of the OMLS, in comparison with the interlayer spacing of the pure OMLS, leading to a shift of the WAXD peak towards the lower value of $2 \Theta$.

Fig. 2a shows the results of WAXD analysis of pure ODA and the corresponding nanocomposite PLA/ODA4 in the range of $2 \Theta=1-10^{\circ}$. The mean interlayer spacing for the ODA powder obtained by WAXD measurement is $2.31 \mathrm{~nm}\left(2 \Theta=3.82^{\circ}\right)$. In the case of PLA/ODA4, a sharp peak is obtained at $2 \Theta=2.78^{\circ}(=3.18 \mathrm{~nm})$. Indicates the formation of intercalated structure but not well ordered because the width of the basal reflection of ODA is decreased sharply after nanocomposite preparation. The width of the WAXD peak, $\beta$ (measured by the full width at half maximum), is inversely proportional to the coherence length of the scattering entities, $D$ and therefore reflects the coherent order of the silicate layers $[54,55]{ }^{2}$ Since the width of the basal spacing of ODA decreased sharply after nanocomposite preparation with PLA, therefore, the coherency of the intercalated silicate layers much higher than that of un-intercalated silicate layers. Thus, we can make conclusion on the basis of WAXD analysis that PLA melt intercalation has strong effect on the layer structure and

\footnotetext{
${ }^{2} D=(\lambda k) /(\beta \cos \theta)$, where $k$ is a constant and generally equal to 0.9 , $\lambda=0.154 \mathrm{~nm}$, and $\theta$ is the WAXD peak position.
} 

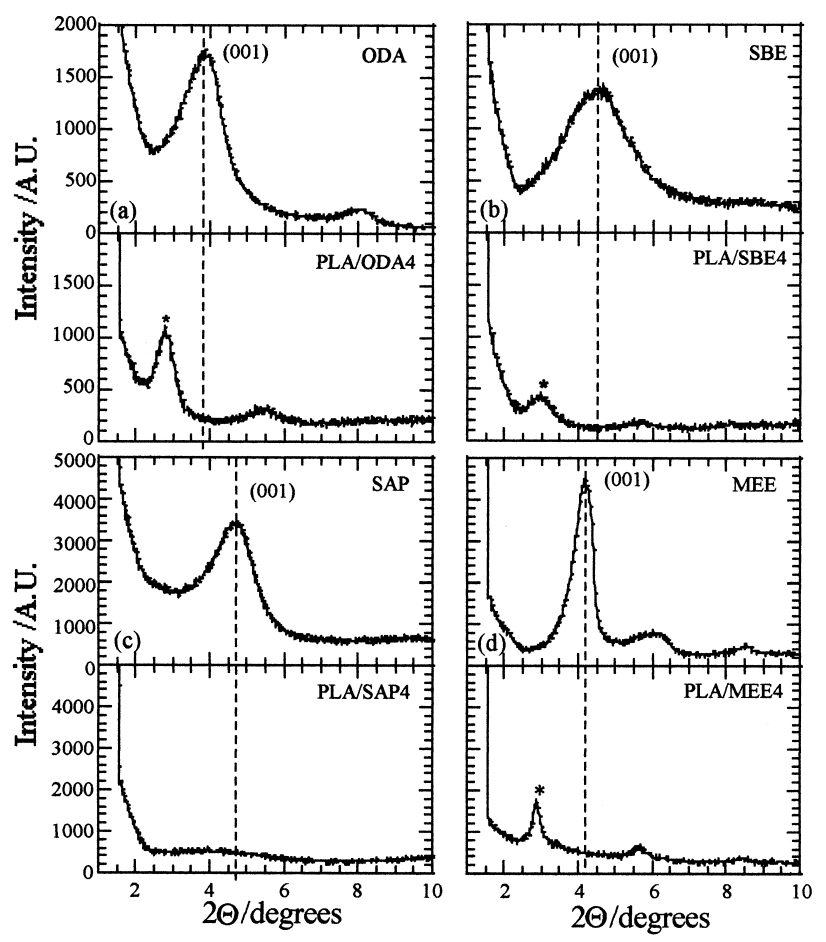

Fig. 2. WAXD patterns of four different types of OMLS and corresponding PLA/OMLS nanocomposites. The dashed line in each figure indicates the location of silicate (001) reflection of OMLS and the asterisk indicates (001) peak for OMLS dispersed in PLA matrix.

drastically change the coherence length of the silicate crystallites.

Fig. 3a is a TEM bright field image of PLA/ODA4, in which dark entities are the cross section of stacked intercalated ODA layers and bright areas are the matrix [56]. ${ }^{3}$ From the TEM image we observed stacked and flocculated silicate layers, which are randomly distributed in the PLA matrix. The WAXD patterns of SBE and the corresponding nanocomposite PLA/SBE4 are shown in Fig. 2b. Fig. $3 \mathrm{~b}$ is a bright-field TEM image of a fully intercalated PLA/SBE4 nanocomposite with comparable magnification to the image of PLA/ODA4 nanocomposite as presented in Fig. 3a.

From the WAXD analyses it is confirmed that the extent of intercalation and at the same time the disruption of the original silicate crystallite structure to a greater extent in the case of PLA/SBE4 than that observed in PLA/ODA4.

In Table 3 we summarized the form factors (see Fig. 1) of various nanocomposites, i.e. average length $\left(L_{\mathrm{LS}}\right)$, thickness $\left(d_{\mathrm{LS}}\right)$ of the dispersed stacked and intercalated silicate layers and the correlation length $\left(\xi_{\mathrm{LS}}\right)$ between them obtained from TEM images. For PLA/ODA $4, L_{\mathrm{LS}}$ and $d_{\mathrm{LS}}$ are in the range of $(450 \pm 200) \mathrm{nm}$ and $(38 \pm 17) \mathrm{nm}$, respectively. On the other hand, PLA/SBE4 exhibit almost same level of stacking of the silicate layers with $L_{\mathrm{LS}}$ of about

\footnotetext{
${ }^{3}$ Since the silicate layers are comprised of heavier elements (AL, Si, O) than the interlayer and surrounding matrix $(\mathrm{C}, \mathrm{H}, \mathrm{N}$, etc.), they appear darker in bright field images.
}

$(200 \pm 25) \mathrm{nm}$ and are more homogeneously distributed in the PLA matrix.

Thus, nanocomposite prepared with ODA leads much more flocculated structure than that obtained with SBE. This is due to the hydroxylated edge-edge interaction of the silicate layers [57]. Owing to the interaction between the silicate particles and PLA matrix, the disk-disk interaction plays an important role in determining the stability of the clay particles, and hence enhancement of mechanical properties of such nanocomposites. On the other hand, $\xi_{\mathrm{LS}}$ value of PLA/SBE4 is in the order of $(80 \pm 20) \mathrm{nm}$, and much lower than the value of PLA/ODA4 (260 \pm 140$) \mathrm{nm})$. So SBE layers are much more delaminated in PLA matrix compared to that of ODA layers.

In contrast to PLA/ODA4 or PLA/SBE4, PLA/SAP4 represents an exfoliated structure. The WAXD pattern of PLA/SAP4 (see Fig. 2c) is almost feature less, only exhibiting a board, and extremely week reflection. In general, many factors other than layer disorder such as intercalate composition, and silicate concentration may contribute to a featureless diffraction [53]. Thus, on the basis of WAXD patterns it is very difficult to make final conclusions about the structure of the nanocomposites exhibiting featureless diffraction pattern. Only the general conclusions about layer order or layer spacing can be inferred. In such cases, TEM is the main tool to determine the exact structure of the nanocomposite.

Fig. $3 c$ is a TEM bright-field image of PLA/SAP4 with comparable magnification to the images of PLA/ODA4 or PLA/SBE4, respectively. Although, the WAXD pattern is featureless, however, stacking of the silicate layers is readily observable, and this is more discernible with high magnification TEM photograph (see Fig. 3d). We believe this behavior comes from very strong interaction between hexadecyltributylphosphonium salt modified silicate surface and PLA matrix in compare to that of alkylammonium salts modified silicate surface and PLA matrix [58].

On the other hand, PLA/MEE4 represents a very sharp WAXD peak (see Fig. 2d), indicates the formation of wellordered intercalated nanocomposite. In Fig. 3e we show a TEM bright-field image of PLA/MEE4. From the TEM image it becomes clear that there are some intercalated stacked ('A' in the TEM Fig.) and disordered or exfoliated MEE layers co-existing in the nanocomposite. Only the stacked intercalated silicate layers are responsible for very sharp WAXD reflection as observed in Fig. 2d, whereas the disordered or exfoliated silicate layers have no periodic stacking and thus remain WAXD silent. This behavior may be intrinsic in MEE-based nanocomposites of polyesters [59]. This type of mixed intercalated/exfoliated structure originates from the chemical and size inhomogeneities of the MEE layers. Typically the large-in lateral size-MEE layers create stacked intercalated structure, whereas the smaller layers tend to exfoliate [47-48].

So in order to study polymer/layered silicate nanocomposite structure, WAXD patterns should be always 

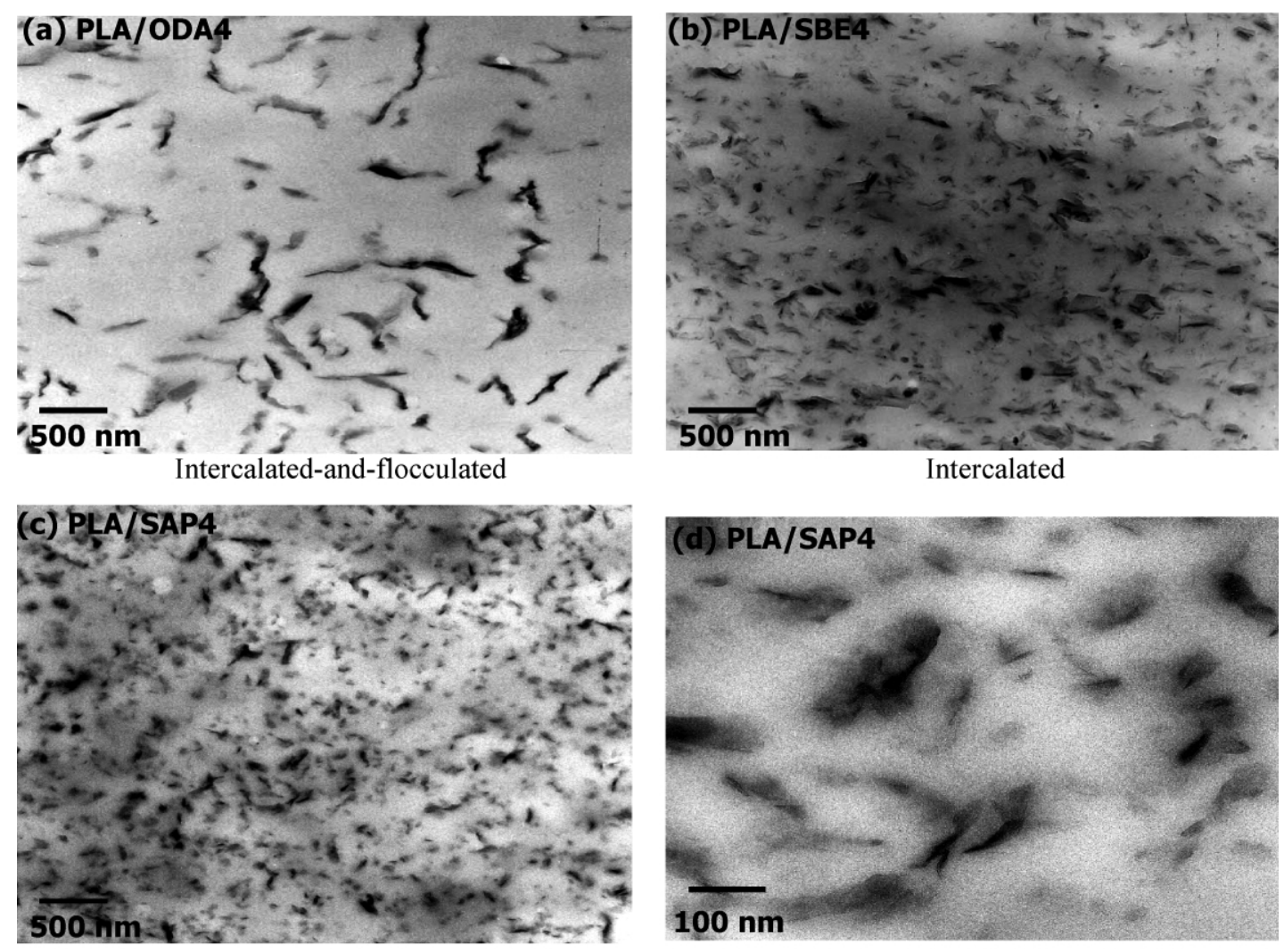

Disordered intercalated or near to exfoliated

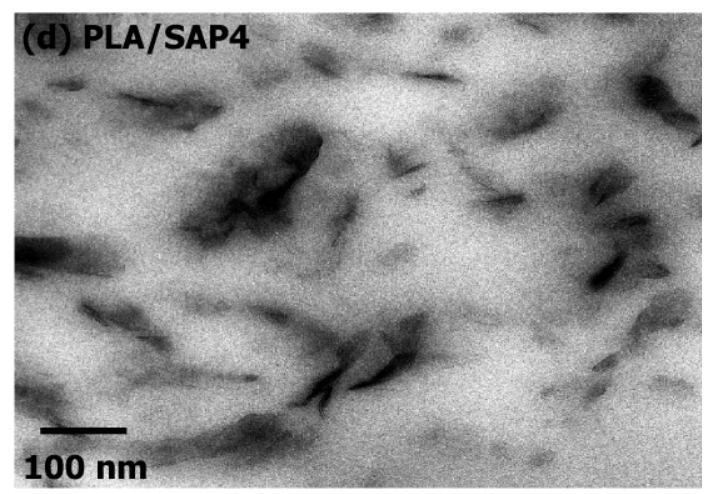

Disordered intercalated or near to exfoliated

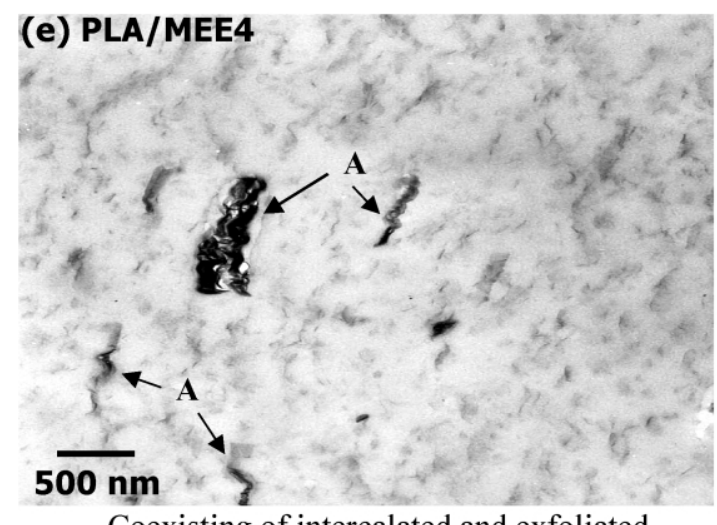

Fig. 3. Bright filed TEM images of various PLA/OMLS nanocomposites. The dark entities are the cross section and or face of intercalated or exfoliated silicate layers and the bright areas are the matrix.

Table 3

Form factors of various nanocomposites obtained from TEM photographs

\begin{tabular}{lllll}
\hline Form factors & PLA/ODA4 & PLA/SBE4 & PLA/SAP4 & PLA/MEE4 \\
\hline$d_{\mathrm{LS}} / \mathrm{nm}$ & $38 \pm 17$ & $36 \pm 9$ & $2-3$ & $1-2$ \\
$L_{\mathrm{LS}} / \mathrm{nm}$ & $450 \pm 200$ & $200 \pm 25$ & $50 \pm 5$ & $275 \pm 25$ \\
$L_{\mathrm{LS}} / d_{\mathrm{LS}}$ & $12-31$ & $14-18$ & $17-27$ & $137-275$ \\
$\xi_{\mathrm{LS}} / \mathrm{nm}$ & $260 \pm 140$ & $80 \pm 20$ & $70 \pm 7$ & $50 \pm 5$ \\
Structure & Intercalated-and-flocculated & Intercalated & Disordered intercalated or near to exfoliated & Coexisting of intercalated and exfoliated \\
\hline
\end{tabular}


accompanied with TEM investigations, since generally there is co-existence of stacked intercalated, disordered or exfoliated structures: thus, a sharp WAXD peak not always confirmed well intercalated structure (in the case of PLA/MEE4), whereas a featureless WAXD pattern may hide large number of stacked silicate layers (in the case of PLA/SAP4). In both cases, the materials properties can be dramatically affected by the structures that are not manifested in the WAXD patterns.

Thus, on the basis of WAXD analyses and TEM observations we can see four different types of nanocomposites were formed. Intercalated-and-flocculated nanocomposites in the case of PLA/ODA4 system, intercalated structure was formed when SBE was used for the nanocomposite preparation, and disordered intercalated or near to exfoliated structure was formed for PLA/SAP4, while coexisting of stacked intercalated/exfoliated nanocomposite was observed in the case of PLA/MEE4.

\subsection{Thermal properties and spherulite morphology}

Fig. 4 shows DSC traces for melt quenched samples of pure PLA and various nanocomposites. Samples were prepared using a hot press. Pure PLA and nanocomposite pellets were first melted at $190{ }^{\circ} \mathrm{C}$ hold for three minutes at the same temperature under $\approx 1.5 \mathrm{MPa}$ pressure, and then quickly quenched between glass plates. From figure the temperature according to the endothermic peak for each sample is considered to be the $T_{\mathrm{g}}$ of PLA. For all samples at $T_{\mathrm{g}}$ there is a step like change, which is due to the enthalpy relaxation [60].

On the other hand, all samples also show exothermic peak that can be correlated to the crystallization of PLA; the corresponding temperature is known as $T_{\mathrm{c}}$. In the case of nanocomposites this peak is sharper and appeared at much lower temperature than that of pure PLA. All OMLS hence seems to enhance the crystallization of PLA. It should be noted here that $T_{\mathrm{c}}$ does strongly depend upon the nature of

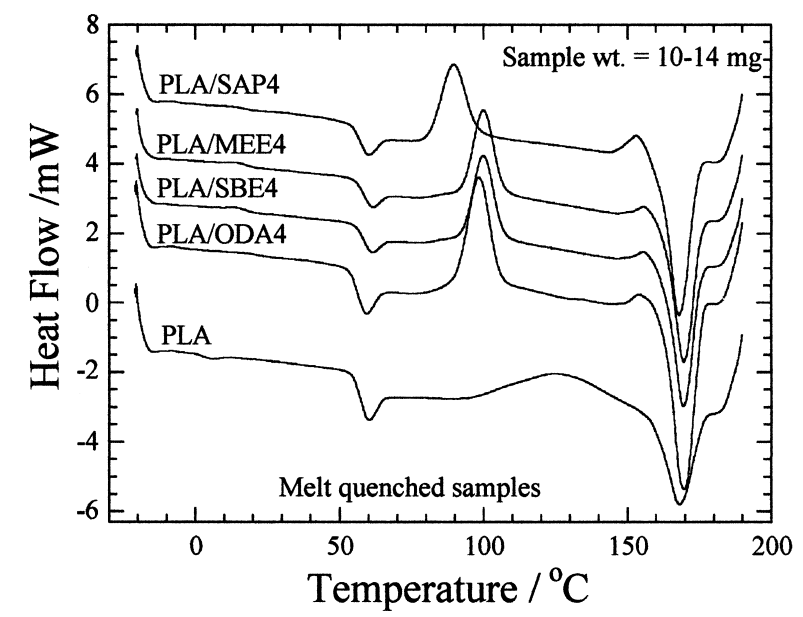

Fig. 4. DSC traces of melt quenched samples of pure PLA and various nanocomposites.
OMLS and is much lower in the case of PLA/SAP4. However, all samples do not show that exothermic peak when annealed at $110^{\circ} \mathrm{C}$ for $1.5 \mathrm{~h}$ before being subjected to DSC analyses. This result indicates PLA matrices were crystallized during heat treatment at $110{ }^{\circ} \mathrm{C}$ for $1.5 \mathrm{~h}$ [61].

POM photographs of pure PLA and various nanocomposites are presented in Fig. 5a. All samples were crystallized at $110^{\circ} \mathrm{C}$ before hand. Pure PLA exhibits well-defined large spherulitic morphology, whereas the sizes of nanocomposite spherulites are significantly smaller. This behavior indicates that the surface of the dispersed OMLS acts as a nucleating agent for PLA crystallization, which is evident from the increase in the number of nuclei causing the formation of smaller crystallite [62]. This decrease in spherulite size is more prominent with PLA/ODA4 and less prominent in PLA/SAP4.

This behavior is more clearly observed in LS patterns (see Fig. 5b), where, for nanocomposites, a large smeared four-leaf-clover pattern is observed compared to the crystallized pure PLA, indicating the formation of large number less well-organized spherulites. From LS patterns, the number of heterogeneous nuclei $N$ can be estimated from a rough approximation. That is, all crystallite are of identical size. The primary nucleation density of the spherulites, i.e. $N$ is given by: [62]

$N=(3 / 4 \pi)\left(D_{\mathrm{m}} / 2\right)^{-3}$

where $D_{\mathrm{m}}$ is the maximum diameter of the spherulite, i.e. the attainable diameter before impingement. The calculated values of $N$ at $110{ }^{\circ} \mathrm{C}$ for pure PLA and various nanocomposites are summarized in Table 2. This phenomenon indicates that the surface of four different types of dispersed OMLS layers act as a nucleating agent for PLA crystallization, which is evident from the increase in number of density of nuclei causing smaller spherulite formation [62], and strongly depends upon the nature of OMLS used for nanocomposites preparation. In spite of low value of $N$, PLA/SAP4 shows the acceleration of crystallization at low $T_{\mathrm{c}}$ range (see Fig. 4), which implies that we have to take account the potentiality of different chain packing with defect structure. On the other hand, WAXD analysis up to $2 \Theta=70^{\circ}$ indicates significant peak shift in crystalline chain-chain distance. The larger inter-chain distance implies the defect-ridden crystallites. This is not the objective of this paper and we will report it separately [63]. Our investigation thus explores the role of OMLS as a nucleating agent for PLA crystallization and crystallization of PLA may thus be controlled by judicious choice of OMLS.

\subsection{Nanocomposites properties}

\subsubsection{DMA}

Dynamic mechanical property measures the response of a given material to an oscillatory deformation (here in tension-torsion mode) as a function of temperature. 
(a)
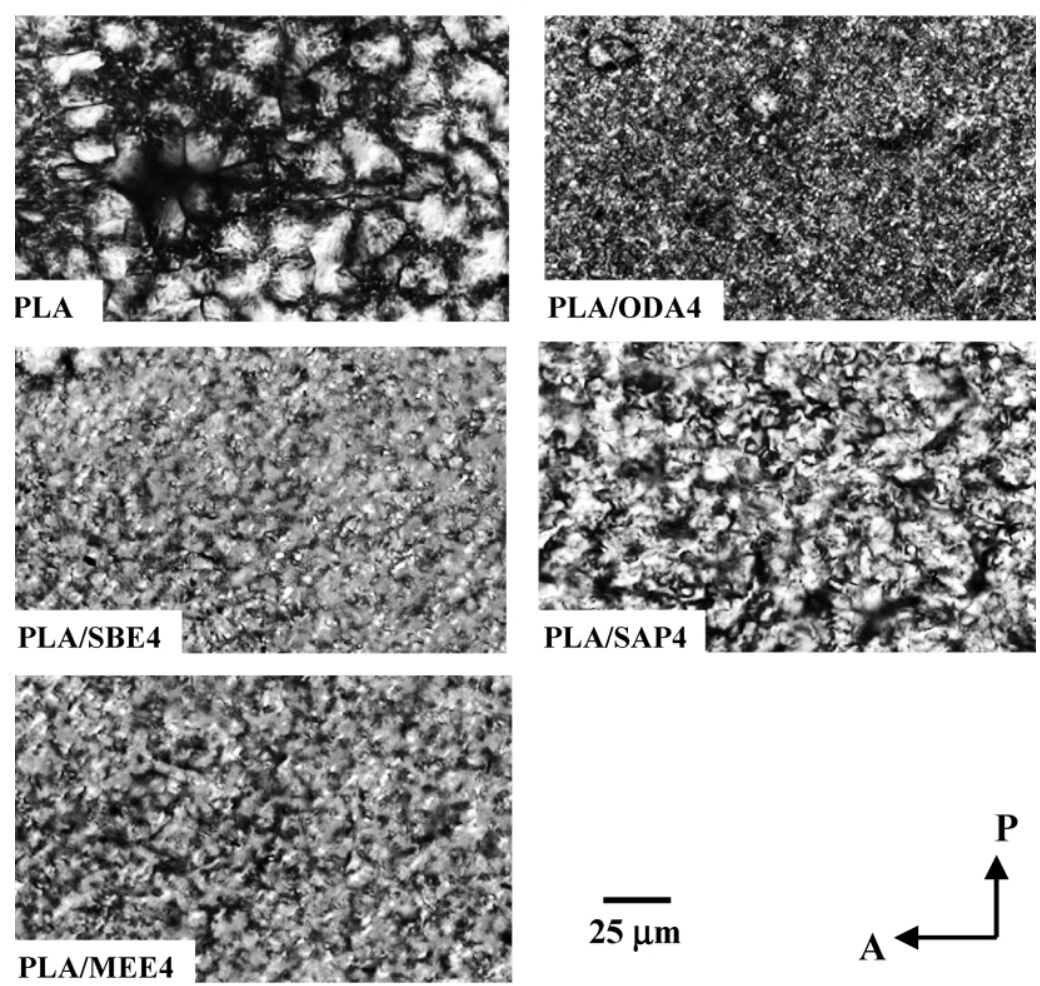

(b)

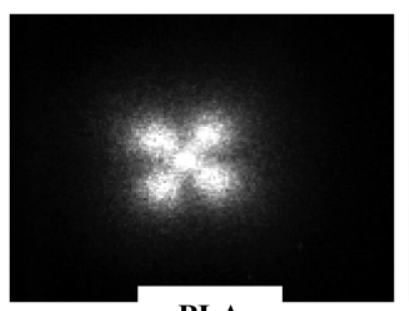

PLA
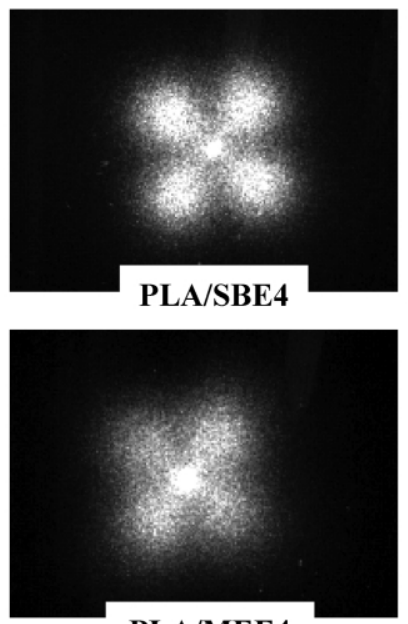

PLA/MEE4

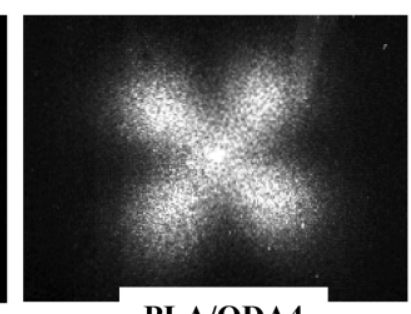

PLA/ODA4

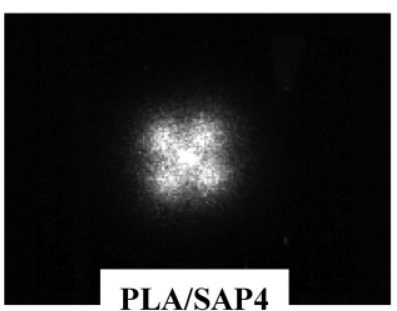

$\underline{q}=\mathbf{2 . 4} \mu \mathbf{m}^{-1}$

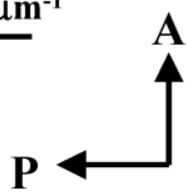

Fig. 5. (a) Polarized optical micrographs of crystallized samples of pure PLA and various nanocomposites isothermally crystallized at $110^{\circ} \mathrm{C}$ for $1.5 \mathrm{~h}$ (b) $\mathrm{Hv}$ light scattering patterns for pure PLA and various nanocomposites isothermally crystallized at $110^{\circ} \mathrm{C}$ for $1.5 \mathrm{~h}$. 

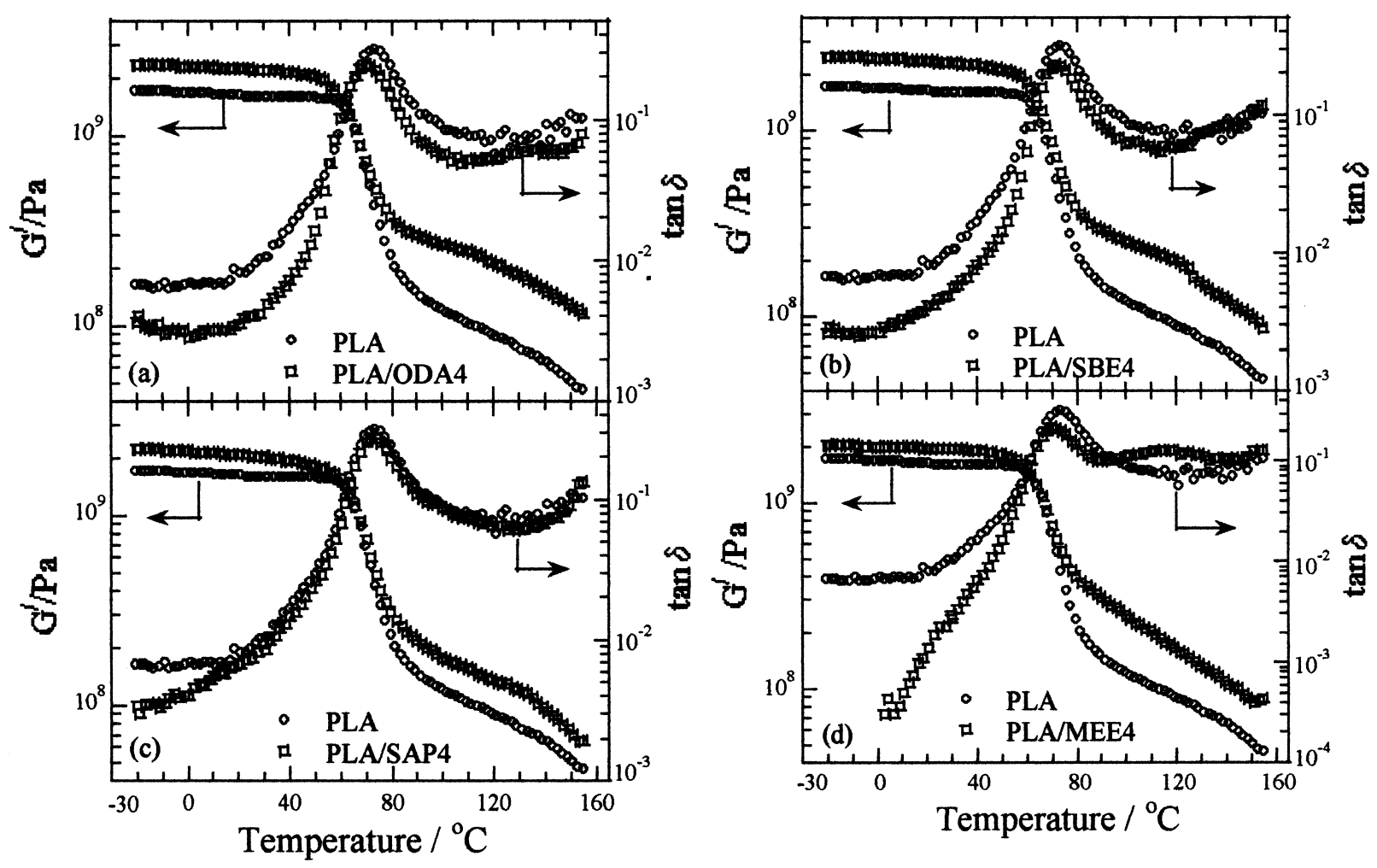

Fig. 6. Temperature dependence of storage modulus $\left(G^{\prime}\right)$ and tan $\delta$ for pure PLA and various nanocomposites.

DMA results are expresses by two main parameters: the $G^{\prime}$, corresponding to the elastic response to the deformation and $\tan \delta$, that is the $G^{\prime \prime} / G^{\prime}$ ratio, useful for determining the occurrence of molecular mobility transitions such as the glass transition temperature.

Here DMA analysis has been studied to track the temperature dependence of $G^{\prime}$ and $\tan \delta$ of pure PLA upon nanocomposite formation with different types of OMLS. Fig. 6 shows the temperature dependence of $G^{\prime}$ and $\tan \delta$ of pure PLA and various nanocomposites. For all nanocomposites, significant enhancement of $G^{\prime}$ can be seen in the investigated temperature range, indicating all OMLS have strong influenced on the elastic properties of the pure PLA. Below $T_{\mathrm{g}}$, the enhancement of $G^{\prime}$ is also clear for all nanocomposites.

At the temperature range of -20 to $0{ }^{\circ} \mathrm{C}$, the increment in $G^{\prime}$ are $37 \%$ for PLA/ODA 4, $45 \%$ for PLA/SBE4, $31 \%$ for PLA/SAP4, and $23 \%$ for PLA/MEE4, compared to that of pure PLA. Furthermore, at the temperature range of $80-$ $90{ }^{\circ} \mathrm{C}$, all nanocomposites exhibit much higher enhancement in $G^{\prime}(96 \%$ for PLA/SBE4, 103\% for PLA/ODA4, and $111 \%$ for PLA/MEE4) than that of pure PLA with the exception of PLA/SAP4 with $45 \%$ increment. This is due to the mechanistic reinforcement by the silicate particles at high temperature $[64,65]$. Above $T_{\mathrm{g}}$, when materials become soft the reinforcement effect of the silicate particles becomes prominent, due to the restricted movement of the polymer chains, and hence strong enhancement of modulus appeared [5]. The restriction of polymer chains movement by the silicate particles is high in the case of PLA/MEE4 because of low value of $\xi_{\text {LS }}$. For this reason PLA/MEE4 shows high increment in $G^{\prime}$ at high temperature range compared to that of other nanocomposites.

On the other hand, at room temperature, PLA/ODA4, PLA/SBE4 and PLA/SAP4 show higher increment in $G^{\prime}$ of 38,44 and $30 \%$ than that of pure PLA, respectively, while that of PLA/MEE4 is only $26 \%$ higher. This increment comes from the extended intercalation in the case of PLA/SBE4 or PLA/ODA4 [62].

However, presence of OMLS does not lead to a significant shift and broadening of the $\tan \delta$ curves for all nanocomposites compared to that of pure PLA. This behavior has been ascribed to the restricted segmental motions at the organic-inorganic interfaces neighborhood of intercalated nanocomposites [44,45].

\subsubsection{Flexural properties and heat distortion test}

Flexural modulus and strength of pure PLA and various nanocomposites measured at $25{ }^{\circ} \mathrm{C}$ are summarized in Table 4 . There is a significant increase in flexural modulus for all nanocomposites except PLA/SAP4 compared to that of pure PLA. The high value of modulus in the case of PLA/MEE4 and low value of modulus in the case of PLA/ SAP4 may be due to the different aspect ratio of dispersed silicate particles in PLA matrix [50]. On the other hand, flexural strength is also remarkably increased in the case of 
Table 4

Comparison of practical materials properties: pure PLA vs. nanocomposites

\begin{tabular}{|c|c|c|c|c|c|}
\hline Practical materials properties & PLA & PLA/ODA4 & PLA/SBE4 & PLA/SAP4 & PLA/MEE4 \\
\hline Flexural modulus/GPa at $25^{\circ} \mathrm{C}$ & 4.84 & 5.66 & 5.57 & 4.5 & 6.11 \\
\hline Flexural strength/MPa at $25^{\circ} \mathrm{C}$ & 86 & 132 & 134 & 93 & 94 \\
\hline Distortion at break $/ \%$ & 1.9 & 3.2 & 3.1 & 1.5 & 1.5 \\
\hline $\mathrm{HDT} /{ }^{\circ} \mathrm{C}$ with $0.98 \mathrm{MPa}$ load & 76 & 94 & 93 & 98 & 93 \\
\hline $\mathrm{O}_{2}$ gas permeability coefficient $/\left(\mathrm{ml} \mathrm{mm} / \mathrm{m}^{2}\right.$ day $\left.\mathrm{MPa}\right)$ & 200 & 172 & 177 & 120 & 71 \\
\hline $\mathrm{O}_{2}$ gas permeability coefficient $/\left(\mathrm{ml} \mathrm{mm} / \mathrm{m}^{2} \text { day } \mathrm{MPa}\right)^{\mathrm{a}}$ & 200 & 180 & 188 & 169 & 68 \\
\hline
\end{tabular}

${ }^{\text {a }}$ Calculated on the basis of Nielsen theoretical equation (Eq. (3) in the text).

PLA/ODA4 or PLA/SBE4 but not so much increase in the case of PLA/MEE4 or PLA/SAP4. This behavior may be due to some brittleness appeared in materials in presence of MEE or SAP silicate particles as revealed by distortion value (see Table 4).

The nano-dispersion of OMLS in the PLA matrix also promotes a higher HDT. We examined the HDT of pure PLA and various nanocomposites with different load conditions but here we report the HDT values with intermediate load of $0.98 \mathrm{MPa}$ (see Table 4). All nanocomposites show significant improvement of HDT in compare to that of pure PLA, and the value increases up to $98{ }^{\circ} \mathrm{C}$ with PLA/SAP4. In the case of PLA/SAP4 studied here, the values of $T_{\mathrm{m}}$ (cf. Table 2) do not change significantly as compared to that of pure PLA. Furthermore, in WAXD analysis up to $2 \Theta=70^{\circ}$, we observed significant shifting of peaks in the crystallized PLA/SAP4 sample. So the strong improvement of HDT in the case of PLA/SAP4 originates from the formation of another crystal structure [66].

\subsection{3. $\mathrm{O}_{2}$ gas permeability}

Layered silicates are believed to increase the gas barrier properties by creating a maze or 'tortuous path' (see schematic illustration in Fig. 7) that retard the progress of gas molecules through the matrix resin $[5,7,8,67,68]$. The $\mathrm{O}_{2}$ gas permeability coefficient for pure PLA and various nanocomposites are presented in Table 4. According to the Nielsen model [69], platelets of length $\left(\cong L_{\mathrm{LS}}\right)$ and width ( $\cong d_{\mathrm{LS}}$ ) of the layered silicate, which are dispersed parallel in polymer matrix, then the tortuosity factor $(\tau)$ can be expressed as

$\tau=1+\left(L_{\mathrm{LS}} / 2 d_{\mathrm{LS}}\right) \phi_{\mathrm{LS}}$

where $\phi_{\mathrm{LS}}$ is the volume fraction of dispersed silicate

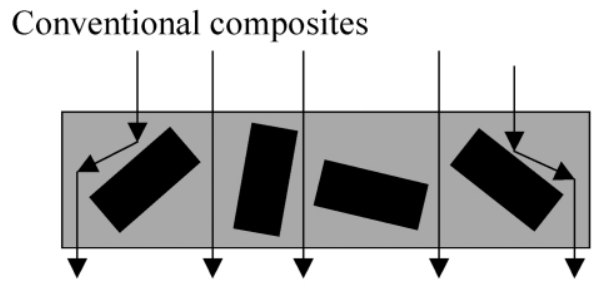

particles. Therefore, the relative permeability coefficient $\left(P_{\mathrm{PLACN}} / P_{\mathrm{PLA}}\right)$ is given by

$\frac{P_{\mathrm{PLACN}}}{P_{\mathrm{PLA}}}=\frac{1}{1+\left(L_{\mathrm{LS}} / 2 d_{\mathrm{LS}}\right) \phi_{\mathrm{LS}}}$

where $P_{\mathrm{PLACN}}$ and $P_{\mathrm{PLA}}$ are the permeability coefficient of nanocomposite and pure PLA, respectively. We consider here PLA/SBE4 system in order to prove the validity of the above model in the case of these nanocomposites, with value of $L_{\mathrm{LS}}=\sim 200 \mathrm{~nm}$ (average value from TEM, Table 3 ), and the value of $d_{\mathrm{LS}}=\sim 36 \mathrm{~nm}$ (average value from TEM image, Table 3). Therefore, the calculated value of $P_{\text {PLACN }} / P_{\text {PLA }}$ for PLA/SBE4 is equal to 0.94 . The experimental value of 0.88 is almost well matched with the above model. The difference between calculated and experimental values may be due to the planer orientation of the dispersed silicate layers in the compress-sheet.

Gas barrier property of nanocomposites primarily depends on two factors: one is the dispersed silicate particles dimension and other is the extent of dispersion of silicate layers in polymer matrix [58]. When the degree of dispersion of layered silicate in the matrix is the same, barrier property completely depends upon the dispersed layered silicate particles dimension that means the aspect ratio.

According to the above theoretical expression as described in Eq. (3), we have calculated the $\mathrm{O}_{2}$ gas transmission coefficient of various nanocomposites using experimentally obtained $L_{\mathrm{LS}} / d_{\mathrm{LS}}$ value as summarized in Table 3. Among the four nanocomposites, the calculated values are almost well match with the experimental values, with the exception of PLA/SAP4 system (see Table 4). The PLA/SAP4 shows higher value of permeability coefficient with comparable aspect ratio to that of other systems.

"Tortuous path" in layered silicate nanocomposites

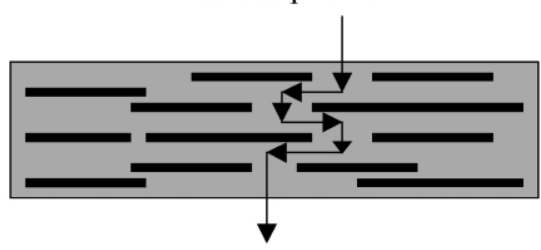

Fig. 7. Schematic illustration of formation of 'tortuous path' in polymer/layered silicate nanocomposites. 
Güsev et al. [70] considered another factor, which is also responsible for the barrier property: changes the local permeability due to the molecular level of transformation in polymer matrix in the presence of silicate layers. This factor is directly related to the molecular level interaction of polymer matrix with the silicate layers. The PLA/SAP4 is a completely disordered intercalated or near to exfoliated system, the favorable interaction between PLA and silicate layers probably due to the formation of phosphonium oxide caused by the reaction between hydroxy edge group of PLA and alkylphosphonium cation. As a result, the barrier property of PLA/SAP4 much higher compared to that of PLA/ODA4 or PLA/SBE4 systems.

\subsubsection{Biodegradation}

Another exciting aspect of this research is the enhanced biodegradability of pure PLA after nanocomposites preparation. A major problem with PLA is the slow rate of degradation as compared to the rate of waste accumulation. Although numerous reports deal with the enzymatic
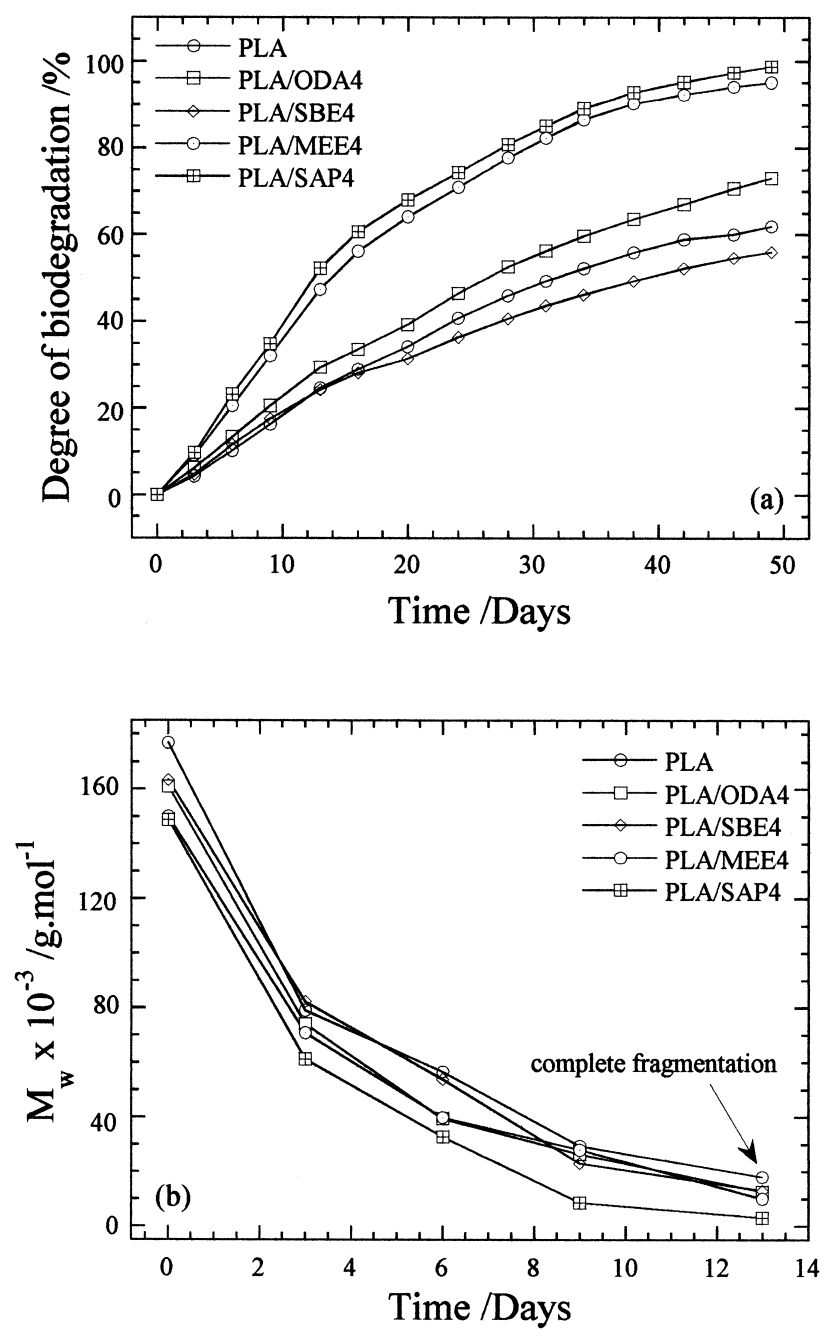

Fig. 8. (a) Degree of biodegradation (i.e. $\mathrm{CO}_{2}$ evolution) and (b) change of matrix $M_{\mathrm{w}}$ of pure PLA and PLA in various nanocomposites under compost. degradation of PLA [71,72] and various PLA blend [73], little is known about the compost degradability of PLA [40, 74,75]. Repirometric tests were carried out to study degradation of PLA matrix in a compost environment at $(58 \pm 2)^{\circ} \mathrm{C}$. We intentionally chose this temperature, as the rate of degradation of pure PLA is very slow at ambient temperature $[40,75]$. Unlike weight loss, which reflects the structural changes in the test sample, $\mathrm{CO}_{2}$ evolution provides an indicator of the ultimate biodegradability, i.e. mineralization, of the samples. Fig. 8a displays the time dependence of the degree of biodegradation of pure PLA and various nanocomposites, indicating that the biodegradability of PLA component in PLA/MEE4 or PLA/SAP4 was enhanced significantly. On the other hand, PLA/ODA4 showed little higher biodegradation rate, while rate of degradation of pure PLA and PLA/SBE4 is almost the same. In general, the degradation of PLA in compost is a complex process involving four main phenomena: (i) water adsorption, (ii) ester bond cleavage and formation of oligomer fragments, (iii) solubilization of oligomer fragments, and finally (iv) diffusion of soluble oligomers by bacteria in terms of $\mathrm{CO}_{2}$ evolution $[40,75]$. Any factor that increases the hydrolysis tendency of the PLA matrix will ultimately control the degradation of PLA.

It is well known that PLA of relatively lower molecular weight may show higher rates of enzymatic degradation because of, for example, a high concentration of accessible chain end groups $[76,77]$. However, the rate of change in molecular weight of pure PLA and PLA in various nanocomposites is almost the same (see Fig. 8b), and also PLA/SBE4 with little low molecular weight show same or rather low rate of degradation compared to that of pure PLA. Therefore, the initial molecular weight cannot be the main factor here to control the biodegradability. Another factor, which control the biodegradability of PLA might be the $\chi_{\mathrm{c}}$ (see Table 2), as an amorphous state is easier to degrade than a crystalline one [71].

These data indicate that the presence of different types of OMLS may thus cause a different mode of attack on the PLA component, which might be due to the presence of different kind of modified salts. The disruption of ester bonds is more facile in the case of MEE or SAP and less facile in case of SBE. Therefore, our investigation explores the role of OMLS as a nano-filler to enhance the rate of biodegradation of pure PLA and we can control the biodegradability of PLA by judicious choice of OMLS [17].

\section{Conclusions}

We have successfully prepared a series of biodegradable PLA/OMLS nanocomposites using a simple melt extrusion of PLA and four different types of OMLS, wherein silicate layers of the OMLS were either intercalated or intercalatedand-flocculated or near to exfoliated or coexistence of intercalated and exfoliated. All nanocomposites exhibited 
remarkable improvements of material properties such as mechanical properties, flexural properties, HDT, oxygen gas permeability, etc. as compared to those of pure PLA, with a simultaneous improvement in biodegradability. Intercalated nanocomposites show very high mechanical properties where as disordered intercalated or near to exfoliated nanocomposites have very high gas barrier property. So depending on the purpose of use we can easily find out the suitable materials and most important thing is that all materials are biodegradable. These concurrent property improvements are well beyond what can be generally achieved through the micro-/macro-composite preparation or chemical modification.

\section{Acknowledgements}

Thanks are due to the Japan Society for the Promotion of Science for awarding a postdoctoral fellowship and a research grant to S. Sinha Ray (No. P02152). We express our appreciation to the reviewer for his constructive and meticulous assessment of the manuscript.

\section{References}

[1] Biswas M, Sinha Ray S. Adv Polym Sci 2001;155:167.

[2] Alexander M, Dubois P. Mater Sci Eng 2000;R28:1.

[3] LeBaron PC, Wang Z, Pinnavaia TJ. J Appl Clay Sci 1999;15:11.

[4] Giannelis EP, Krishnamoorti R, Manias E. Adv Polym Sci 1999;138: 107.

[5] Sinha Ray S, Okamoto M. Prog Polym Sci 2003; in press.

[6] Giannelis EP. Appl Organomet Chem 1998;12:675.

[7] Xu R, Manias E, Snyder AJ, Runt J. Macromolecules 2001;34:337.

[8] Bharadwaj RK. Macromolecules 2001;34:9189.

[9] Messersmith PB, Giannelis EP. J Polym Sci Part A: Polym Chem 1995;33:1047.

[10] Yano K, Usuki A, Okada A, Kurauchi T, Kamigaito O. J Polym Sci Part A: Polym Chem 1993;31:2493.

[11] Kojima Y, Usuki A, Kawasumi M, Fukushima Y, Okada A, Kurauchi T, Kamigaito O. J Mater Res 1993;8:1179.

[12] Gilman JW. Appl Clay Sci 1999;15:31.

[13] Gilman JW, Jackson CL, Morgan AB, Harris R. Chem Mater 2000;12: 1866.

[14] Bourbigot S, Le Bras M, Dabrowski F, Gilman JW, Kashiwagi T. Fire Mater 2000;24:201.

[15] Gilman JW, Jackson CL, Morgan AB, Harris Jr R, Manias E, Giannelis EP, Wuthenow M, Hilton D, Phillips SH. Chem Mater 2000;12:1866.

[16] Sinha Ray S, Yamada K, Okamoto M, Ueda K. Nano Lett 2002;2: 1093.

[17] Sinha Ray S, Yamada K, Okamoto M, Ogami A, Ueda K. Macromol Mater Eng 2003;288:203.

[18] Vaia RA, Jandt KD, Kramer EJ, Giannelis EP. Macromolecules 1995; 28:8080.

[19] Krishnamoorti R, Vaia RA, Giannelis EP. Chem Mater 1996;8:1728.

[20] Chen JS, Poliks MD, Ober CK, Zhang Y, Wiesner U, Giannelis EP. Polymer 2002;43:4895.

[21] Maiti P, Okamoto M. Macromol Mater Eng 2003;288:440.

[22] Cao G, Mallouk TE. J Solid State Chem 1991;94:59.

[23] Pillion JE, Thompson Me E. Chem Mater 1991;3:777.
[24] Kanatzidis MG, Wu CG, Marcy HO, deGroot DC, Kannewurf CR. Chem Mater 1990;2:222.

[25] Mehrotra V, Giannelis EP. Solid State Commun 1991;77:155.

[26] Aranda P, Ruiz-Hitzky E. Chem Mater 1992;4:1395.

[27] Massersmith PB, Stupp SI. J Mater Res 1992;7:2599.

[28] Vaia RA, Ishii H, Giannelis EP. Chem Mater 1993;5:1994.

[29] Hasewaga N, Okamoto H, Kato M, Usuki A. J Appl Polym Sci 2000; 78:1981.

[30] Hasewaga N, Okamoto H, Kato M, Tsukigase A, Usuki A. Macromol Mater Eng 2000;280:76.

[31] Okamoto M, Nam PH, Maiti P, Kotaka T, Hasegawa N, Usuki A. Nano Lett 2001;1:295.

[32] Okamoto M, Nam PH, Maiti P, Kotaka T, Nakayama T, Takada M, Ohsima M, Usuki A, Hasegawa N. Nano Lett 2001;1:503.

[33] Vaia RA, Giannelis EP. Macromolecules 1997;30:8000. and references cited therein.

[34] Tsuji H, Ikada Y. J Appl Polym Sci 1998;67:405.

[35] Martin O, Averous L. Polymer 2001;42:6209.

[36] Kim SH, Han YK, Kim YH, Hong SI. Macromol Chem 1991;193: 1623.

[37] Kricheldorf HR, Serra A. Polym Bull 1985;14:497.

[38] Kricheldorf HR, Berl M, Scharngal N. Macromolecules 1988;21:286.

[39] Nijenhuis AJ, Grijpma DW, Pennings AJ. Macromolecules 1992;25: 6419.

[40] Lunt J. Polym Degrad Stab 1998;59:145.

[41] Qi F, Hanna MA. Industrials Crops Pdts 1999;10:47.

[42] Gu JD, Gada M, Kharas G, Eberiel D, McCarthy SP, Gross RA. Polym Mater Sci Eng 1992;67:351.

[43] Ogata N, Jimenez G, Kawai H, Ogihara TJ. Polym Sci Part B: Polym Phys 1997;35:389.

[44] Sinha Ray S, Maiti P, Okamoto M, Yamada K, Ueda K. Macromolecules 2002;35:3104.

[45] Sinha Ray S, Yamada K, Okamoto M, Ogami A, Ueda K. Composite Interfaces 2003;10. in press.

[46] Sinha Ray S, Okamoto K, Yamada K, Okamoto M. Nano Lett 2002;2: 423.

[47] Sinha Ray S, Yamada K, Okamoto M, Ogami A, Ueda K. Macromol Rapid Commun 2002;23:943.

[48] Sinha Ray S, Yamada K, Okamoto M, Ogami A, Ueda K. Chem Mater 2003; $15: 1456$.

[49] Sinha Ray S, Yamada K, Okamoto M, Ueda K. Polymer 2003;44:857.

[50] Nam PH, Maiti P, Okamoto M, Kotaka T, Hasegawa N, Usuki A. Polymer 2001;42:9633.

[51] Fischer EW, Sterzel HJ, Wegner G, Kolloid ZZ. Polymer 1973;25: 980.

[52] Okamoto M, Kubo H, Kotaka T. Macromolecules 1998;31:4223.

[53] Vaia RA, Jandt KD, Kramer EJ, Giannelis EP. Chem Mater 1996;8: 2628.

[54] Dritis VA, Tchoubar C, X-ray diffraction by disordered lamellar structures, vol. 99. New York: Springer; 1990. p. 21-22.

[55] Cullity BD. Principles of X-ray diffraction. Rading, MA: AddisonWesley; 1978.

[56] Klimentidis RE, Mackinnon IDR. Clays Clay Miner 1986;34:155.

[57] Okamoto M, Morita S, Kim YH, Kotaka T, Tateyama H. Polymer 2001;42:1201.

[58] Maiti P, Yamada K, Okamoto M, Ueda K, Okamoto K. Chem Mater 2002; $14: 4654$.

[59] Okamoto K, Sinha Ray S, Okamoto M, unpublished results.

[60] Wunderlich B, Macromolecular physics, vol. 2. New York: Academic Press; 1976. p. 363.

[61] Eling B, Gogolewski S, Pennings AJ. Polymer 1977;23:1587.

[62] Maiti P, Nam PH, Okamoto M, Usuki A, Hasegawa N. Macromolecules 2002;35:2042.

[63] Nam JY, Sinha Ray S, Okamoto M. Macromolecules 2003; in press.

[64] Sinha Ray S, Okamoto K, Okamoto MJ. Nanosci Nanotech 2002;2: 471. 
[65] Sinha Ray S, Okamoto K, Okamoto M. Macromolecules 2003;36: 2355.

[66] Manias E, Touny A, Wu L, Strawhecker K, Lu B, Chung TC. Chem Mater 2001;13:3516.

[67] Usuki A, Kawasumi M, Kojima Y, Okada A, Kurauchi T, Kamigaito O. J Mater Res 1993;8:1185.

[68] Messersmith PB, Giannelis EP. Chem Mater 1994;6:1719.

[69] Nielsen LJ. Macromol Sci Chem 1967;A1(5):929.

[70] Güsev AA, Lusti HR. Adv Mater 2001;13:1641.

[71] Reeve MS, MaCarthy SP, Downey MJ, Gross RA. Macromolecules 1994;27:825. and references cited therein.
[72] Iwata T, Doi Y. Macromolecules 1998;31:2461.

[73] Fambri L, Pegoretti A, Fenner R, Incardona SD, Migliaresi C. Polymer 1997;38:79.

[74] Hakkarainen M, Karlsson S, Albertsson AC. Polymer 2000;41: 2331.

[75] Drumright RE, Gruber PR, Henton DE. Adv Mater 2000;12:1841.

[76] Kawai F. CRC Crit Rev Biotechnol 1987;6(3):273.

[77] Taino T, Fukui T, Shirakura Y, Saito T, Tomita K, Kaiho T, Masamune S. Eur J Biochem 1982;124:71. 